\title{
Tracing mixing in stars: new beryllium observations of the open clusters NGC 2516, Hyades, and M 67^
}

\author{
S. Randich ${ }^{1}$, F. Primas ${ }^{2}$, L. Pasquini ${ }^{2}$, P. Sestito ${ }^{1}$, and R. Pallavicini ${ }^{3}$ \\ 1 INAF/Osservatorio Astrofisico di Arcetri, Largo E. Fermi 5, 50125 Firenze, Italy \\ e-mail: randich@arcetri.astro.it \\ 2 European Southern Observatory, Garching bei München, Germany \\ 3 INAF/Osservatorio Astronomico di Palermo, Piazza del Parlamento 1, 90134 Palermo, Italy
}

Received 9 August 2006 / Accepted 11 April 2007

\begin{abstract}
Context. Determinations of beryllium abundance in stars, together with lithium, provide a key tool to investigate the so far poorly understood extra-mixing processes at work in stellar interiors.

Aims. We measured Be in three open clusters, complementing existing Be surveys, and aiming at gathering a more complete empirical scenario of the evolution of Be as a function of stellar age and temperature.

Methods. We analyzed VLT/UVES spectra of members of NGC 2516, the Hyades, and M 67 to determine their Be and Li abundances. In the first two clusters we focused on stars cooler than $5400 \mathrm{~K}$, while the M 67 sample includes stars warmer than $6150 \mathrm{~K}$, as well as two subgiants and two blue stragglers. We also computed the evolution of Be for a $0.9 M_{\odot}$ star based on standard evolutionary models. Results. We find different behaviours for stars in different temperature bins and ages. Stars warmer than $6150 \mathrm{~K}$ show Be depletion and follow a Be vs. Li correlation, while Be is undepleted in stars in the $\sim 6150-5600 \mathrm{~K}$ range. NGC 2516 members cooler than $5400 \mathrm{~K}$ have not depleted any $\mathrm{Be}$, while older Hyades of similar temperature show some depletion. Be is severely depleted in the subgiants and blue stragglers.

Conclusions. The results for warm stars are in agreement with those of previous studies, supporting the hypothesis that mixing in this temperature regime is driven by rotation. The same holds for the two subgiants that have evolved from the "Li gap". This mechanism is instead not the dominant one for solar-type stars. We show that Be depletion of cool Hyades cannot simply be explained by the effect of increasing depth of the convective zone. Finally, the different Be content of the two blue stragglers suggests that they have formed by two different processes (i.e., collisions vs. binary merging).
\end{abstract}

Key words. stars: abundances - stars: evolution - stars: interiors - open clusters and associations: individual: M 67 open clusters and associations: individual: NGC 2516 - open clusters and associations: individual: Hyades

\section{Introduction}

Beryllium $(\mathrm{Be})$ is easily burned in stellar interiors at temperatures above $\sim 3.5 \mathrm{MK}$; as a consequence, any process that is able to mix surface material with internal Be-depleted material results in surface Be depletion. Since the more fragile lithium (Li) is destroyed at temperatures above $\sim 2.5 \mathrm{MK}$, measurements of both elements in the same star allow us to investigate how deep mixing extends in the stellar interior and to put constraints on the various extra-mixing processes introduced in evolutionary models to explain the complex $\mathrm{Li}$ behavior. Several unexpected results related to the depletion of $\mathrm{Li}$ in stars in fact have been found, which reveal our limited understanding of the physics acting in the interiors of stars.

Focusing on late-F and early G-type stars, different empirical evidences contradict the predictions of standard models (those including convection only). In particular, observations of $\mathrm{Li}$ in open clusters of different ages have shown that these stars deplete Li during the main sequence (MS), in spite of the fact that their convective zones do not reach deep enough in the stellar interior to meet the zone of $\mathrm{Li}$ destruction (e.g., Randich 2006 , and references therein); the factor of $\sim 100 \mathrm{Li}$ depletion

* Based on observations collected at ESO-VLT, Paranal Observatory, Chile, Programme numbers 65.L-0427, 68.D-0491, 69.D-0454. measured in the Sun itself indeed represents a long standing puzzle. Moreover, a factor of $\sim 10$ scatter in $\mathrm{Li}$ is observed in the solar-age, solar metallicity cluster M 67 (Spite et al. 1987; García López et al. 1988; Pasquini et al. 1997; Jones et al. 1999), indicating that otherwise similar stars (i.e., stars with the same age, temperature, and metallicity) may be affected by different amounts of Li depletion, again in disagreement with standard models. This dispersion implies that $\mathrm{Li}$ depletion must be affected by an additional parameter besides mass, age, and chemical composition.

As to warmer early-F stars, since the 80s' Boesgaard and collaborators pointed out the presence of a so-called "Li gap" or "Li dip" among hot MS stars in the Hyades cluster, namely a sharp decrease of $\mathrm{Li}$ in a narrow $(\sim 300 \mathrm{~K})$ effective temperature $\left(T_{\text {eff }}\right)$ range around $6600 \mathrm{~K}$ (Boesgaard \& Tripicco 1986). The gap is present in several other clusters of the Hyades age or older (Balachandran 1995, and references therein), is weak or absent in the 120 Myr Pleiades (Pilachowski et al. 1987; Boesgaard et al. 1988), but has been detected in the slightly older M 35 (160 Myr - Steinhauer \& Deliyannis 2004), suggesting that it develops at ages below $200 \mathrm{Myr}$.

In order to explain the unexpected MS Li depletion of F and early-G type stars, a number of models and extra-mixing processes have been proposed, which include mass loss (Swenson \& Faulkner 1992), diffusion (Michaud 1986; Chaboyer et al. 1995), 
meridional circulation (Charbonnel \& Talon 1999, and references therein), angular momentum loss, and rotationally driven mixing (Schatzman \& Baglin 1991; Pinsonneault et al. 1992; Deliyannis \& Pinsonneault 1997), gravity waves (García López \& Spruit 1991; Montalban \& Schatzmann 2000), tachocline (Brun et al. 1999; Piau et al. 2003), and combinations of these (Charbonnel \& Talon 2005). Note that models including rotational mixing would in principle be able to explain the scatter il $\mathrm{Li}$ in $\mathrm{M} \mathrm{67,} \mathrm{with} \mathrm{initial} \mathrm{angular} \mathrm{momentum} \mathrm{being} \mathrm{the} \mathrm{additional}$ parameter affecting Li depletion. Since models including different extra-mixing processes predict distinct behaviours of $\mathrm{Li}$ vs. Be with age and with mass, the last few years have witnessed a flourishing of Be observations among field and cluster stars. The availability of state-of-the art high resolution spectrographs with high near-UV efficiency has made it possible to observe not only bright stars in close-by clusters, but also fainter members of more distant, old clusters.

These Be surveys have allowed constraining the mechanism responsible for the gap; more specifically, several studies have now evidenced the presence of a correlation between $\mathrm{Li}$ and Be among cluster and field stars on the cool/red side of the gap ( $T_{\text {eff }}$ between $\sim 6600$ and $6300 \mathrm{~K}$ ), providing strong support to rotationally induced mixing (e.g., Deliyannis et al. 1998; Boesgaard et al. 2004a,b). As for cooler stars in older clusters, Randich et al. (2002; hereafter R02) found that solar-type members $\left(T_{\text {eff }} \sim 6150-5800 \mathrm{~K}\right)$ of M 67 and the 2 Gyr old IC 4651 have not depleted any Be and thus do not follow any Li-Be correlation. This finding allowed them to rule out a number of models; in particular, at variance with warmer stars on the red side of the gap, present models including rotationally induced mixing do not seem to well reproduce the observed Be vs. Li pattern for stars cooler than $6150 \mathrm{~K}$ in M 67: whereas they predict that at old ages these stars should have depleted some Be (a factor 2-3), no depletion is instead observed.

In this work we present new Be measurements in three open clusters: the young NGC 2516 (150 Myr), the Hyades (600 Myr), and the old M 67 (4.5 Gyr). In NGC 2516 and the Hyades we mostly concentrated on cool MS stars ( $T_{\text {eff }}$ below $5400 \mathrm{~K}$ ), the reason being that warmer stars in both the Hyades and other young clusters (such as the Pleiades and Alpha Per) have been extensively studied in the past by Boesgaard and collaborators (see Boesgaard et al. 2004b, and references therein). In M 67 we instead observed stars warmer than in the R02 sample, as well as two subgiants and two blue stragglers. Since the upper turnoff of M 67 coincides with the cool edge of the Li gap, subgiants are supposed to be evolved from objects that were in the gap (Balachandran 1995); their $\mathrm{Li}$ and Be abundances are therefore important to constrain the gap evolution at old ages. As to blue stragglers, their light element content might provide insights into their formation mechanism. In summary, when joining our sample with available Be cluster data from the literature, we have a coverage of the $T_{\text {eff }}$ interval from the $\mathrm{Li}$ gap down to $5100 \mathrm{~K}$ for three groups of stars, with ages of $0.15,0.6$ and $4.5 \mathrm{Gyr}$. Based on this sample, our goal is to obtain a more complete empirical picture of the evolution of $\mathrm{Be}$ (and $\mathrm{Be}$ vs. $\mathrm{Li}$ ) as a function of stellar age and temperature.

\section{Sample stars and observations}

The sample includes four members of NGC 2516, four members of the Hyades and eight members of M 67. The observations were carried out in Service mode using UVES on VLT/Kueyen (Dekker et al. 2000) during the period November 2001-March 2002. All stars were observed using the same set-up; namely, UVES was operated in Dichroic Mode with Cross Dispersers \#1 and \#3 in the Blue and Red arms, respectively. Such a combination allowed us to cover the spectral ranges from $\sim 3115$ to $3940 \AA$ in the blue and from $\sim 4780$ to $6810 \AA$ in the red. The $15 \mu \mathrm{m}$ pixels and the use of a 1 arcsec wide slit (projecting onto 4 pixels) and CCD binning $2 \times 2$ and $1 \times 1$ in the blue and red yielded resolving powers $R \sim 40000$ and $R \sim 45000$, respectively. The spectra were reduced by the ESO Quality Control Group using the UVES pipeline implemented in the MIDAS context. After careful inspection, we decided that the quality of the reduced spectra delivered by ESO did not require a new reduction of the raw frames; therefore, we just coadded spectra taken during different exposures, after applying the correction for radial velocity shifts and normalization. Total exposure times range between 3600-10800 s, depending on the star magnitude. The final $S / N$ ratios per resolution element are in the intervals $\sim 20$ and 80 in the Be region and $\sim 120$ and 350 in the Li region. The sample stars together with the log of the observations and achieved $S / N$ ratios are listed in Table 1.

\section{Abundance and error analysis}

Effective temperatures ( $T_{\text {eff's }}$ ) for the majority of M 67 stars were retrieved from Jones et al. (1999, see also Randich et al. 2006) who, in turn, had derived them from dereddened $B-V$ colors using the calibration of Soderblom et al. (1993). We determined $T_{\text {eff's }}$ for the two M 67 members not included in Jones et al. sample (S1034 and S1239) and for NGC 2516 and Hyades stars in the same fashion. For M 67 a reddening $E(B-V)=0.04$ was used, following Jones et al., while we assumed $E(B-V)=$ 0.01 and 0.12 for the Hyades and NGC 2516. Adopted $T_{\text {eff }}$ values are listed in the second column of Table 2. A surface gravity $\log g=4.5$ was assumed for MS stars in NGC 2516 and the Hyades, while for M 67 we used the spectroscopic values of Randich et al. (2006). Microturbulence was derived from $\log g$ and $T_{\text {eff }}$ based on the relationship $\xi=3.2 \times 10^{-4}\left(T_{\text {eff }}-6390\right)-$ $1.3(\log g-4.16)+1.7 \mathrm{~km} \mathrm{~s}^{-1}($ Nissen 1981).

Both $\mathrm{Li}$ and $\mathrm{Be}$ analyses were performed in the same way as in $\mathrm{R} 02$. Li abundances were derived by means of equivalent widths $(E W \mathrm{~s})$ of the $\mathrm{Li}$ I $6707.8 \AA$ feature and using Kurucz's WIDTH9 (Kurucz 1993) code and model atmospheres. Our spectral resolution, together with the low rotational velocity of all sample stars, allowed us to resolve the Li line from the nearby Fe I $6707.4 \AA$ feature and hence there was no need to correct measured Li $E W s$ for its contribution. EWs and derived Li abundances are listed in Cols. 3 and 4 of Table 2. Errors associated to our $\mathrm{Li}$ abundances range between 0.08 and 0.17 dex. These combine the uncertainties in the $E W$ measurement (as reported in Col. 3 of the table, and which include also the uncertainty due to the placement of the continuum) and in the stellar parameters (i.e. how much the $\mathrm{Li}$ abundance is affected by changes in the stellar parameters); as well known, the dominant source is the high sensitivity of Li to the effective temperature $(0.08$ dex per $100 \mathrm{~K})$. Uncertainties in the stellar parameters were taken from Randich et al. (2006) for M 67 and are as follows: $\pm 70 \mathrm{~K}$ in $T_{\text {eff }}, \pm 0.25$ in $\log g, \pm 0.15 \mathrm{~km} \mathrm{~s}^{-1}$ in $\xi$. We assumed the same uncertainties for the Hyades and NGC 2516. For a more detailed breakdown of all uncertainties affecting Li determination, we refer the reader to Charbonnel \& Primas (2005).

As mentioned, Be abundances were determined using the same spectral synthesis recipes as in R02 because we would like to combine both samples for our final discussion. Namely, we synthesized a region of $\approx 5 \AA$, around the Be II doublet 
Table 1. Sample stars and $\log$ of the observations. Listed $S / N$ ratios are per resolution element.

\begin{tabular}{lrlrrrr}
\hline \hline ID & $V$ & $(B-V)_{0}$ & Obs. Date & $\begin{array}{c}\text { Exp. time } \\
(\mathrm{s})\end{array}$ & $S / N @$ Be & $S / N @$ Li \\
\hline M 67 S990 & 13.43 & 0.525 & Main sequence stars \\
M 67 S995 & 12.76 & 0.521 & Feb. 3 2002 & 7200 & 35 & 150 \\
M 67 S998 & 13.06 & 0.518 & Jan. 7 2002 & 5400 & 40 & 200 \\
M 67 S2205 & 13.14 & 0.534 & Mar. 1, 4 2002 & 7200 & 40 & 175 \\
Hyades vB21 & 9.14 & 0.81 & Dec. 3 2001 & 1200 & 75 & 350 \\
Hyades vB109 & 9.40 & 0.81 & Dec. 23 2001 & 1800 & 80 & 350 \\
Hyades vB182 & 9.01 & 0.83 & Dec. 21 2001 & 1200 & 80 & 350 \\
Hyades vB187 & 9.01 & 0.75 & Nov. 29 2001 & 1200 & 80 & 350 \\
NGC 2516 CTIO-2 & 14.20 & 0.79 & Dec. 19, 23 2001 & 10800 & 20 & 150 \\
NGC 2516 CTIO-3 & 14.30 & 0.82 & Dec. 20, 22 2001 & 10800 & 20 & 150 \\
NGC 2516 CTIO-6 & 13.62 & 0.66 & Dec. 30 2001 & 9600 & 30 & 180 \\
NGC 2516 DK213 & 14.01 & 0.76 & Dec. 28 2001 & 5400 & 20 & 120 \\
& \multicolumn{7}{c}{ Blue stragglers and subgiants } \\
M 67 S997 & 12.13 & 0.410 & Feb. 24 2002 & 3600 & 50 & 210 \\
M 67 S2204 & 12.89 & 0.417 & Mar. 8, Apr. 6 2002 & 5400 & 40 & 180 \\
M 67 S1034 & 12.65 & 0.567 & Apr. 15, 16 2002 & 4800 & 35 & 200 \\
M 67 S1239 & 12.75 & 0.692 & Mar. 7, 8 2002 & 5400 & 30 & 190 \\
\hline
\end{tabular}

Numbering for M 67 from Sanders (1977).

Numbering for the Hyades from van Bueren (1952).

Numbering for NGC 2516 from Jeffries et al. (1998).

Table 2. Effective temperatures, equivalent widths of the Li I $6707.8 \AA$ line, and derived $\mathrm{Li}$ and Be abundances. These are in the usual notation $\log n(\mathrm{X})=\log N(\mathrm{X}) / N(\mathrm{H})+12$. Note that a positive offset of $\sim 0.1-0.2$ dex in $\log n(\mathrm{Be})$ might be present for stars cooler than $5400 \mathrm{~K}$ - see text.

\begin{tabular}{lcccr}
\hline \hline Star & $\begin{array}{c}T_{\text {eff }} \\
(\mathrm{K})\end{array}$ & $\begin{array}{c}E W(\mathrm{Li}) \\
(\mathrm{mA})\end{array}$ & $\log n(\mathrm{Li})$ & $\log n(\mathrm{Be})$ \\
& \multicolumn{5}{c}{ Main sequence stars } \\
M 67 S990 & 6191 & $45 \pm 4$ & $2.63 \pm 0.07$ & $0.96 \pm 0.13$ \\
M 67 S995 & 6210 & $17 \pm 2$ & $2.10 \pm 0.08$ & $0.91 \pm 0.13$ \\
M 67 S998 & 6223 & $44 \pm 2$ & $2.64 \pm 0.06$ & $0.91 \pm 0.13$ \\
M 67 S2205 & 6156 & $11 \pm 3$ & $1.86 \pm 0.16$ & $0.81 \pm 0.13$ \\
Hyades vB21 & 5141 & $\leq 3$ & $\leq 0.27$ & $0.85 \pm 0.21$ \\
Hyades vB109 & 5141 & $5 \pm 1$ & $0.49 \pm 0.11$ & $0.80 \pm 0.21$ \\
Hyades vB182 & 5079 & $10 \pm 1$ & $0.73 \pm 0.07$ & $0.80 \pm 0.21$ \\
Hyades vB187 & 5339 & $17 \pm 1$ & $1.27 \pm 0.06$ & $0.80 \pm 0.21$ \\
NGC 2516 CTI0-2 & 5238 & $147 \pm 3$ & $2.69 \pm 0.06$ & $1.16 \pm 0.21$ \\
NGC 2516 CTI0-3 & 5110 & $174 \pm 4$ & $2.80 \pm 0.07$ & $1.04 \pm 0.21$ \\
NGC 2516 CTIO-6 & 5659 & $124 \pm 5$ & $2.93 \pm 0.07$ & $1.21 \pm 0.21$ \\
NGC 2516 DK213 & 5305 & $108 \pm 2$ & $2.41 \pm 0.06$ & $1.06 \pm 0.21$ \\
\multicolumn{5}{c}{ Blue stragglers and subgiants } \\
M 67 S997 & 6700 & $\leq 5$ & $\leq 1.91$ & $0.31 \pm 0.25$ \\
M 67 S2204 & 6668 & $\leq 5$ & $\leq 1.89$ & $-0.20 \pm 0.29$ \\
M 67 S1034 & 5969 & $\leq 3$ & $\leq 1.14$ & $0.12 \pm 0.13$ \\
M 67 S1239 & 5477 & $8 \pm 1$ & $1.12 \pm 0.10$ & $0.11 \pm 0.21$ \\
\hline
\end{tabular}

$(\lambda=3130.420 \AA$ and $\lambda=3131.064 \AA)$, with Kurucz's model atmospheres and SYNTHE code (Kurucz 1993) and with the line list assembled and thoroughly tested by Primas et al. (1997). Because of the severe blanketing present at these wavelengths, and because we are working with solar (or over-solar) metallicity stars, the normalization of the spectra is critical. Since no line-free region is available around the Be II lines, we normalized our observed spectra by dividing them for the highest number of counts in each spectrum, in a 15-20 ̊̊region centered on the Be II doublet; this step was performed before multiple spectra for a given object were co-added. The spectra were then further shifted (by a few \%, on average) to match the computed synthetic spectra, but the overall continuum shape was

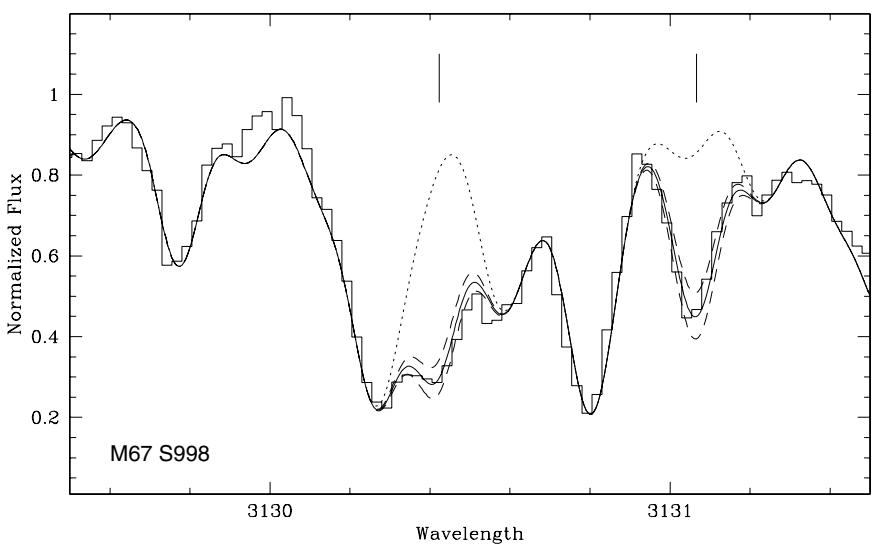

Fig. 1. The observed spectrum of the M 67 star S998 in the Be region with four spectrum syntheses overplotted: our best-fit synthesis (continuous line), two syntheses computed respectively with a variation of the Be content of \pm 0.15 dex (dashed lines) and one synthesis computed without beryllium (dotted line). The two vertical bars identify the components of the Be II doublet.

maintained. A few examples of spectrum syntheses are presented in Figs. 1-4, where we show our best-fit synthesis, two syntheses computed by varying the Be content by a fixed amount, and one synthesis computed without Be.

Our final $\log n(\mathrm{Be})$ (as derived with 1D model atmospheres and including a predicted $\mathrm{Fe} \mathrm{I}$ line in our list of transitions) and associated uncertainties (see below) are listed in the last column of Table 2.

As a final remark, we note that Randich et al. (2006) derived spectroscopic values of surface gravity for their sample stars in M 67 somewhat different from those assumed by R02; for this reason we carried out a new analysis of the two stars (S988 and S994) whose new $\log g$ values differed from the old ones by more than 0.25 dex (the assumed error in $\log g$ ). New and old abundances for these stars are listed in Table 3. In the following we will consider the new abundances derived in this work. 


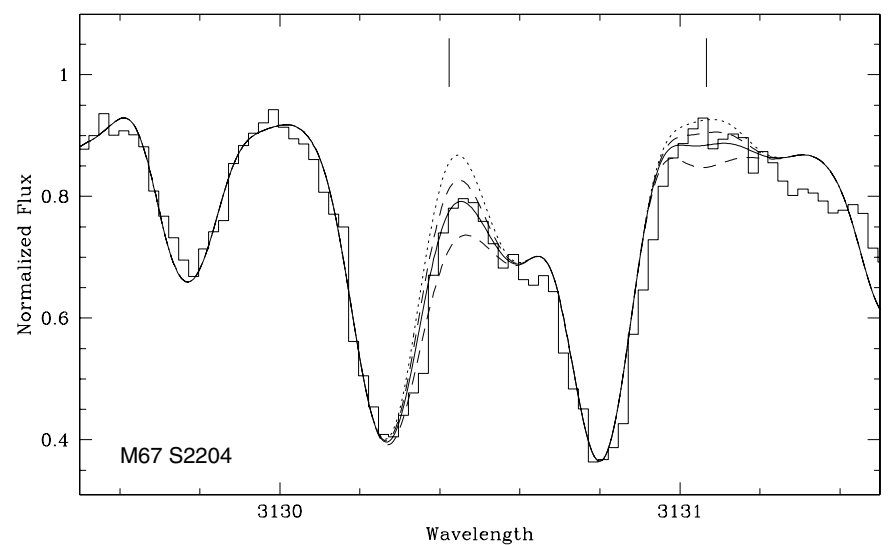

Fig. 2. Same as Fig. 1, but for the M 67 star S2204 and for a variation in the Be content of \pm 0.3 dex.

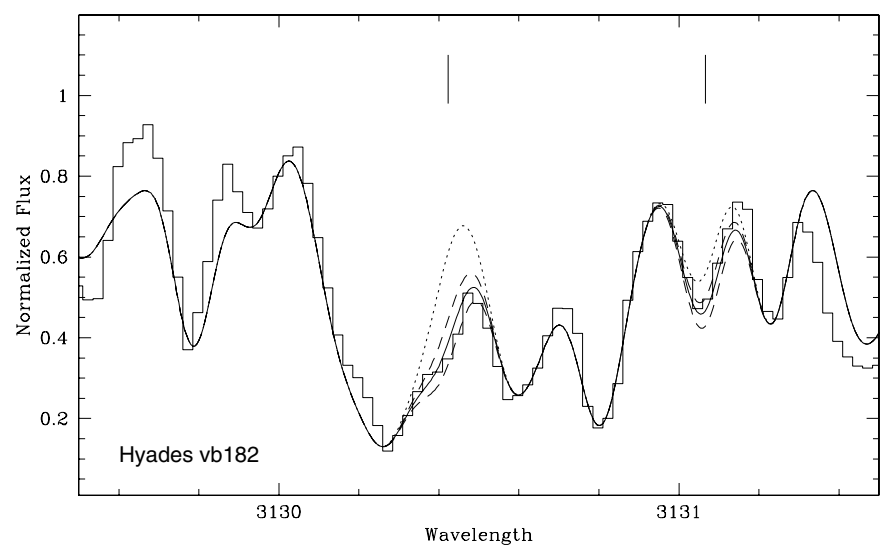

Fig. 3. Same as Fig. 1, but for the Hyades star vB182 and for a variation in the Be content of \pm 0.25 dex.

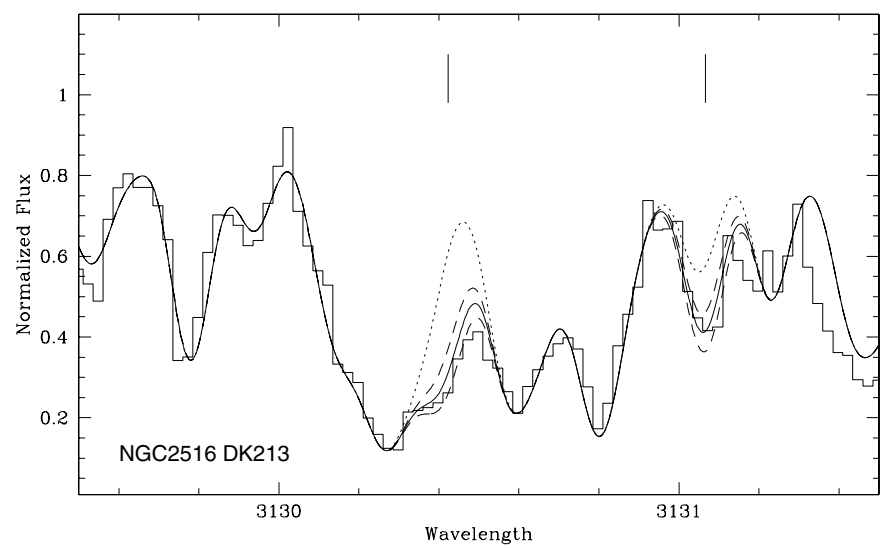

Fig. 4. Same as Fig. 1, but for the NGC 2516 star DK213 and for a variation in the Be content of $\pm 0.25 \mathrm{dex}$.

\subsection{Errors in Be abundances}

\subsubsection{Random uncertainties}

In order to estimate the uncertainty associated to our Be abundances, we computed the sensitivity of the derived $\mathrm{Be}$ abundances to changes of the stellar parameters and of the placement of the continuum. Our final errors do not include the uncertainty related to the atomic physics of the Be transitions (the $\log g f$ values are supposedly known with high accuracy) or the absolute error on the model atmospheres, which is difficult to properly estimate. Because our sample includes stars in different
Table 3. New (present analysis) and old (R02) beryllium abundances for S988 and S994 already analyzed by R02.

\begin{tabular}{llcccc}
\hline \hline Star & $T_{\text {eff }}$ & $\log g_{\text {old }}$ & $\log n(\mathrm{Be})_{\text {old }}$ & $\log g_{\text {new }}$ & $\log n(\mathrm{Be})_{\text {new }}$ \\
\hline S988 & 6153 & 4.5 & 0.88 & 4.1 & 0.65 \\
S994 & 6151 & 4.5 & 1.16 & 4.1 & 0.86 \\
\hline
\end{tabular}

Table 4. Sensitivity of Be abundances to $T_{\text {eff }}, \log g, \xi$, [Fe/H], and placement of the continuum, for three stars of our sample.

\begin{tabular}{cccc}
\hline \hline & S998 & S2204 & vB182 \\
\hline$T_{\text {eff }}(\mathrm{K})$ & 6222 & 6668 & 5079 \\
$\pm 70 \mathrm{~K}$ & $\pm 0.03 \mathrm{dex}$ & $\pm 0.07 \mathrm{dex}$ & $\pm 0.05 \mathrm{dex}$ \\
$\log g$ & 4.0 & 4.0 & 4.5 \\
\pm 0.25 & $\pm 0.10 \mathrm{dex}$ & $\pm 0.08 \mathrm{dex}$ & $\pm 0.17 \mathrm{dex}$ \\
$\xi\left(\mathrm{km} \mathrm{s}^{-1}\right)$ & 1.50 & 1.35 & 0.84 \\
$\pm 0.15 \mathrm{~km} \mathrm{~s}^{-1}$ & 0.00 & 0.00 & $\pm 0.05 \mathrm{dex}$ \\
{$[\mathrm{Fe} / \mathrm{H}](\mathrm{dex})$} & 0.00 & 0.00 & 0.13 \\
$\pm 0.05 \mathrm{dex}$ & $\mp 0.03 \mathrm{dex}$ & $\mp 0.10 \mathrm{dex}$ & $\mp 0.05 \mathrm{dex}$ \\
$\pm 3 \%$ cont. & $\mp 0.04 \mathrm{dex}$ & $\mp 0.25 \mathrm{dex}$ & $\mp 0.08 \mathrm{dex}$ \\
\hline$\sigma_{\text {tot }}$ & $\pm 0.13 \mathrm{dex}$ & $\pm 0.29 \mathrm{dex}$ & $\pm 0.21 \mathrm{dex}$ \\
\hline & & &
\end{tabular}

evolutionary stages, we performed our sensitivity analysis for three stars characterized by different stellar parameters: S998, vB182, and S2204. We chose S998 as representative of our MS M 67 sample, vB182 as representative of the cooler MS stars, and S2204 as one of the hottest stars in our sample with very little Be left. Because the metallicity of these clusters is rather well determined, we assumed an uncertainty of $\pm 0.05 \mathrm{dex}$ on $[\mathrm{Fe} / \mathrm{H}]$. For the placement of the continuum, we estimated that $\pm 3 \%$ is representative of this uncertainty. This number was derived by normalizing our observed spectra with a different method, i.e. using the computed synthetic spectra as the main reference to be matched. A summary of our Be error analysis is provided in Table 4, where the numbers reported in the last row $\left(\sigma_{\text {tot }}\right)$ are the square root of the sum in quadrature of all factors. We note that when the dependence on some stellar parameters was not perfectly symmetric (when increasing or decreasing that parameter by the chosen fixed amount), we always took the average as the representative value.

\subsubsection{Systematic uncertainties}

We remind from R02 that the list of lines we have used includes a predicted line (i.e., not fully identified in the laboratory) of neutral iron at $\lambda=3131.043 \AA$ which is needed in order to obtain a satisfactory fit of the $\lambda=3131.064 \AA \mathrm{Be}$ line in the solar spectrum. Our analysis of the Sun yields a photospheric solar Be abundance $\log n(\mathrm{Be})_{\odot}=1.11$, in excellent agreement with the value found by Chmielewski et al. (1975). The atomic characteristics of this line were constrained by Primas et al. (1997) on a large sample of stars, spanning a wide range of stellar parameters (in temperature, gravity and metallicity). It is important to note, however, that García López et al. (1995) proposed an alternative solution to the unsatisfactory fit of the redder component of the Be doublet, i.e. an increase of the oscillator strength of the Mn I line at $\lambda=3131.037 \AA$ by $+1.5 \mathrm{dex}$ (from the value of -1.725 reported in the Kurucz linelist). They found that such line becomes the dominant blending transition of the redder component of the Be doublet for stars with effective temperature below $5200 \mathrm{~K}$, which prevented them from making a full detection of $\mathrm{Be}$ in their lowest temperature stars. Because of this finding and of the fact that, at solar (or above solar) 
metallicity, the predicted line used by Primas et al. (1997) was tested only down to $\sim 5400 \mathrm{~K}$ (and our current sample reaches instead $5079 \mathrm{~K}$ ), we decided to run a few more tests and to compare the effect of the two proposed solutions, especially for the lowest temperature stars.

First of all, we confirmed that both solutions do not have any important effect for stars above $5400 \mathrm{~K}$, except for allowing a better fit of the blue wing of the $\lambda=3131.066 \AA$. As we tested lower effective temperatures, we found that the two solutions differ by 0.2 dex in the final derived $\mathrm{Be}$ abundance in the 5000-5100 K range, while in the 5250-5400 K interval the difference amounts to $0.15-0.10 \mathrm{dex}$, with our solution always yielding lower Be abundances. In other words, for stars below $5400 \mathrm{~K}$ the strength of the predicted Fe I line is larger than that of the the $\mathrm{Mn} \mathrm{I}$ and the difference increases with decreasing temperature. Note, however, that our proposed solution still allows us to measure Be abundances down to the lowest $T_{\text {eff }}$ in our sample. Whereas there is no solid argument in favour of one solution with respect to the other, depending on the solution one decides to follow, the lowest temperature stars may end up having slightly different abundances, with the offset depending on the temperature. For this reason we are not able to derive a definitive absolute Be abundance and thus to quantify the exact amount of Be depletion suffered by Hyades members in our sample; nevertheless the relative abundances of NGC 2516 and Hyades stars with similar temperatures suggest that the latter have undergone some depletion.

\section{Results}

\subsection{Comparison with previous studies}

Our Li abundances for most stars are in very good agreement with the values derived in previous studies (Jeffries et al. 1998, for NGC 2516; Thorburn et al. 1993, for the Hyades; Jones et al. 1999 and Balachandran 1995, for M 67). For one star only, CTIO-6 in NGC 2516, we derive an abundance somewhat lower than (but given the errors still consistent with) the value of Jeffries et al. (1998); namely, we obtain $\log n(\mathrm{Li})=2.93$ to be compared with value $\log n(\mathrm{Li})=3.04$. Note however that Jeffries et al. (1998) measured a much higher $E W(212 \mathrm{m \AA})$, which in our abundance scale would correspond to $\log n(\mathrm{Li}) \sim 3.4$. Also, we detected the Li line in the spectra of two stars for which only upper limits were available in the literature (S2205: $\log n(\mathrm{Li})_{\text {pres. }}=1.86$ vs. $\log n(\mathrm{Li})_{\text {lit. }} \leq 2.34-$ Jones et al. 1999; S1239: $\log n(\mathrm{Li})_{\text {pres. }}=1.12$ vs. $\log n(\mathrm{Li})_{\text {lit. }} \leq 1.1-$ Balachandran 1995) and were able to put more stringent upper limits on Li abundances for three stars: namely S997 and S2204 $\left(\log n(\mathrm{Li})_{\text {pres. }} \leq 1.91\right.$ vs. $\log n(\mathrm{Li})_{\text {lit. }} \leq 2.46 ; \log n(\mathrm{Li})_{\text {pres. }} \leq 1.89$ vs. $\log n(\mathrm{Li})_{\text {lit. }} \leq 2.36$ - Shetrone $\&$ Sandquist 2000) and S1034 $\left(\log n(\mathrm{Li})_{\text {pres. }} \leq 1.14\right.$ vs. $\log n(\mathrm{Li})_{\text {lit. }} \leq 1.6-$ Balachandran 1995).

Our study provides the first determination of Be for all the sample stars, but vB21; this star was included in the study of García López et al. (1995) who measured $\log n(\mathrm{Be})=0.90 \pm 0.25$, in agreement with our own determination $(\log n(\mathrm{Be})=0.85 \pm$ $0.21)$.

\subsection{Be vs. $T_{\text {eff }}$}

In Fig. 5 we plot $\log n(\mathrm{Be})$ (upper panel) and $\log n(\mathrm{Li})$ (lower panel) as a function of $T_{\text {eff }}$ for our sample stars and the sample of R02. The present M 67 sample includes four new stars warmer
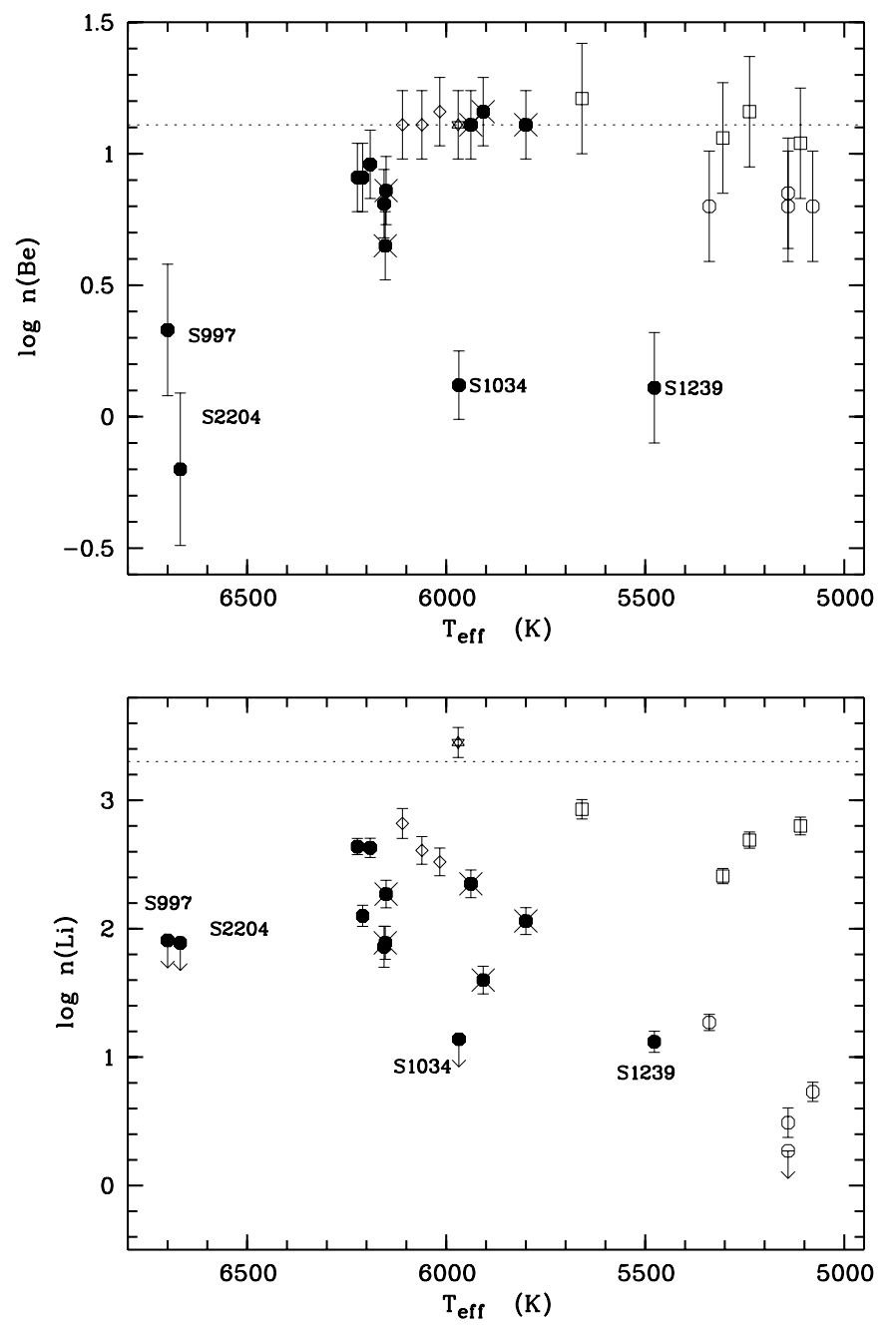

Fig. 5. Upper panel: beryllium abundances $(\log n(\mathrm{Be})$, in the usual $\log$ arithmic scale where $\log n(\mathrm{H})=12$ ) as a function effective temperature $\left(T_{\text {eff }}\right)$ for our sample stars plus the sample of R02. Symbols are as follows: M 67: filled circles (present sample) and crossed filled circles (R02); NGC 2516: open squares; Hyades: open circles; IC 4651 (R02): open diamonds; IC 2391 (R02): open star symbol. Lower panel: same as upper panel, but $\mathrm{Li}-\log n(\mathrm{Li})$ - abundances are plotted. Names of M 67 stars out of the MS are indicated in both panels. Horizontal lines denote initial $\mathrm{Be}$ and $\mathrm{Li}$ abundances in our scales.

than $6150 \mathrm{~K}$, but no cooler cluster members; therefore the conclusions of R02 for solar-type stars remain unchanged: independently of their absolute Be abundance, almost all the stars in the range $5600 \leq T_{\text {eff }} \leq 6150 \mathrm{~K}$ show, within the errors, the same relative abundance, in spite of the different levels of $\mathrm{Li}$ depletion (see lower panel of Fig. 5). On the other hand, stars warmer than $6150 \mathrm{~K}$ both in the present and in the R02 samples have lower abundances, suggesting that they have suffered a certain amount of Be depletion. Note that, as discussed in detail in Sect. 5.1 below, we consider the abundance of SHJM2, a member of the 55 Myr old cluster IC 2391, as representative of the value of the initial Be abundance in our scale.

As to stars cooler than $5400 \mathrm{~K}$ - note that our sample does not include stars with $T_{\text {eff }}$ in the range $5400-5600 \mathrm{~K}-$, Be abundances of NGC 2516 members are consistent with no depletion in spite of the factor $\sim 2-8 \mathrm{Li}$ depletion. The significantly more Li-poor Hyades have lower Be abundances: the average value of the four Hyades stars is $\sim 0.25 \mathrm{dex}$ (or a factor 1.8 ) below the average of the three NGC 2516 stars cooler than $5400 \mathrm{~K}$. This 
result would not change if the abundances were derived assuming the solution proposed by García López et al. (1995 - see discussion in Sect. 3).

Finally, both the two blue stragglers (S997 and S2204) and the two evolved M 67 members are characterized by significant amounts of $\mathrm{Li}$ and Be depletion.

\section{Discussion}

\subsection{Initial beryllium abundance}

As discussed by $\mathrm{R} 02$, the $\mathrm{Be}$ abundance of the young star SHJM2 in IC $2391(\log n(\mathrm{Be})=1.11)$, as well as our value for the photospheric solar Be $(\log n(\mathrm{Be})=1.11)$, are about a factor of two lower than the meteoritic abundance $(\log n(\mathrm{Be})=1.42-$ Anders \& Grevesse $1989^{1}$ ). Abundances for stars in the present study and in R02 are all below this value. Also, maximum Be abundances similar to ours are found by García López et al. (1995) for the Hyades and by Santos et al. (2004) for their solar metallicity stars; both studies derive a Be abundance for the Sun very close to our determination. IC 2391 has a solar metallicity (Randich et al. 2001) and thus its initial Be abundance is most likely the same as for the solar system. The difference between our $\log n(\mathrm{Be})$ values for the Sun and SHJM2 and the meteoritic Be abundance suggests that either a) both the Sun and SHJM2 have depleted a factor of two Be; or b) they have not undergone any depletion, the difference with the meteoritic value is due to systematic effects in the adopted analysis, and the $\log n(\mathrm{Be})$ value of SHJM2 is indicative of the initial Be abundance in our scale. We mention in passing that the discrepancy between the solar photospheric $\mathrm{Be}$ and meteoritic abundance has been known since long time and it has been discussed in several works.

Hypothesis a) and, specifically, the fact that SHJM2 has depleted some Be, would imply that Be burning occurs during the pre-main sequence (PMS) phases, at variance with the observational evidence of no PMS Li depletion for stars in this $T_{\text {eff }}$ range (Randich et al. 2001, and references therein); it would indeed be hard to explain how this star depleted some Be while maintaining its initial Li. For this reason, we regard possibility a) as very unlikely and favor hypothesis b) above. At the same time, since the metallicities of M 67 and NGC 2516 are also solar (Randich et al. 2006; Jeffries et al. 1998), it is unlikely that these clusters had different initial Be abundances due to Galactic evolutionary effects. We conclude therefore that all the sample stars with $\log n(\mathrm{Be})$ consistent with the abundance of SHJM2, including the Sun, have most likely not undergone any Be depletion. A strong support to this hypothesis is given by the analysis of Balachandran \& Bell (1998) of the solar Be who showed that, taking into account an additional amount of UV opacity, needed to solve the inconsistency between solar oxygen abundance derived from IR and UV lines, accounts perfectly for the missing $\mathrm{Be}$ and makes the discrepancy between the solar photospheric $\mathrm{Be}$ and the meteoritic value disappear. The conclusion that the photospheric solar Be is consistent with the meteoritic one was also reached by Asplund et al. (2004); our assumption hence appears fully justified. We finally mention that a higher value of the solar Be abundances was derived by Boesgaard et al. (2003: $\left.\log n(\mathrm{Be})_{\odot}=1.30\right)$, by using 2002 version of MOOG which includes all of the Kurucz opacity edge; also, for undepleted cluster stars in their sample, they derive maximum abundances

1 A slightly lower value, $\log n(\mathrm{Be})=1.38 \pm 0.08$, has been recently reported by Asplund et al. (2005).

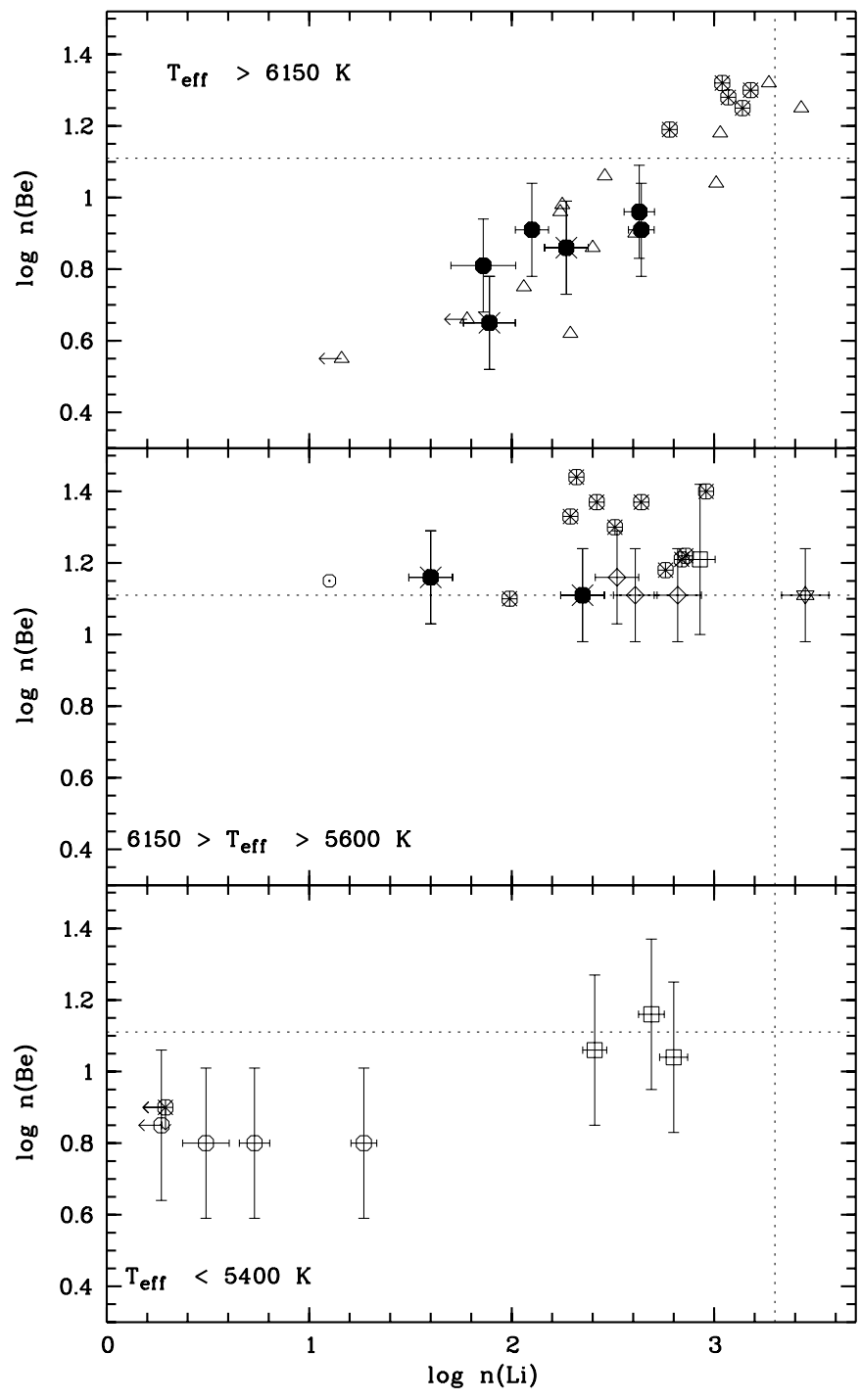

Fig. 6. $\log n(\mathrm{Be})$ as a function of $\log n(\mathrm{Li})$ for our sample stars, the sample of R02, and the Hyades from Boesgaard et al. (2004a) and García López et al. (1995). Upper panel: stars warmer than $6150 \mathrm{~K}$. Middle panel: stars with $5600 \leq T_{\text {eff }}<6150 \mathrm{~K}$; Lower panel: stars cooler than $5400 \mathrm{~K}$. M 67 members out of the MS are not plotted in the figure. The Sun is also shown in the middle panel. Symbols are the same as in Fig. 5; Hyades from the literature, not plotted in Fig. 5, are represented here as open circles with stars (in all panels for $T_{\text {eff }} \leq 6300 \mathrm{~K}$ ) and open triangles (upper panel, stars with $T_{\text {eff }}>6300 \mathrm{~K}$ ). Horizontal and vertical dashed lines denote initial $\mathrm{Be}$ and $\mathrm{Li}$ abundances in our scales.

higher than ours (and in particular than that of SHJM2). These differences suggest the presence of an offset between the abundance scales.

\subsection{Be and Li depletion}

In Fig. 6 we plot $\log n(\mathrm{Be})$ as a function of $\log n(\mathrm{Li})$ for our sample stars, the sample of R02, and the Hyades (from Boesgaard et al. 2004a and García López et al. 1995). In the upper, middle, and lower panels we show stars in three temperature ranges: namely, $6300>T_{\text {eff }} \geq 6150 \mathrm{~K}, 5600 \leq T_{\text {eff }}<6150 \mathrm{~K}$, and $T_{\text {eff }}<5400 \mathrm{~K}$. This subdivision broadly corresponds to the different regimes of Be depletion seen in Fig. 5; specifically, stars that show both $\mathrm{Li}$ and Be depletion, stars that show Li depletion, but no Be depletion, stars that show Li depletion and 
may show Be depletion. Whereas the precise boundaries between these three intervals might be slightly different, it is important to subdivide the sample stars in subgroups since, as we discuss below, they likely correspond to different dominant mixing processes. In the upper panel we show for comparison also Hyades stars on the red side of the Li gap. We finally note that our sample does not include stars with $T_{\text {eff }}$ between 5400 and 5600; this $T_{\text {eff }}$ range is thus not represented in the figure.

The figure evidences distinct behaviors for stars in the three subsamples. Namely, the middle panel, which includes only stars from R02 plus the Hyades, clearly indicates the absence of a Be vs. Li correlation for solar-type stars in the 6150-5600 K range in both old and young clusters: they span two orders of magnitude in Li abundances, they have different ages and metallicities, but they share the same Be content. This in turn implies that the mixing mechanism responsible for MS Li depletion in this temperature range does not extend deep enough to cause also Be depletion. On the other hand, the enlarged sample with respect to R02 shows that a correlation between Li and Be abundances is present for warmer stars in $M$ 67; these stars rather well fit into the Be vs. Li trend for the Hyades on the red side of the Li gap.

Finally, the bottom panel and, in particular the difference between Be abundances of the Hyades and NGC 2516 members, suggest that in the Hyades cool stars Li depletion is accompanied by some amount of Be depletion. It would be not formally correct to claim a Be-Li correlation for these stars, since NGC 2516 members cover a large range in $\mathrm{Li}$ abundance, but have not depleted any Be; also, Hyades stars themselves show different amounts of Li depletion, but the same Be content. Nevertheless, the figure shows that, in this $T_{\text {eff }}$ regime, at variance with solartype stars, but similarly to warmer objects $\left(T_{\text {eff }}>6150 \mathrm{~K}\right)$, the mechanism responsible for Li depletion can reach deep enough to cause also Be depletion. We stress again that, whereas the absolute Be abundances of the coolest sample stars in the figure would be higher had we used the solution of García López et al. (1995), the relative depletion would remain the same.

\subsection{Cool stars: Be as a function of age}

García López et al. (1995) already found evidence of Be depletion for two cool Hyades members, while Santos et al. (2004) detected Be depletion in several old field stars cooler than about $5600 \mathrm{~K}$. Our results not only reinforce the conclusion that cool stars might deplete Be, but most importantly, the comparison between stars with similar temperatures and different ages allows us to put constraints on the age when Be depletion starts on the MS.

In Fig. 7 we plot $\log n(\mathrm{Be})$ as a function of $T_{\text {eff }}$ for NGC 2516, the Hyades (present, García López et al. 1995 and Boesgaard et al. 2004a, samples), the Pleiades from Boesgaard et al. (2003), and old field stars from Santos et al. (2004). Note that the original sample of Santos et al. is mostly composed by stars older than $1 \mathrm{Gyr}$ and we do not plot in the figure the four young stars in their sample. The figure shows that Be depletion starts being present below $\sim 5600 \mathrm{~K}$ : for $T_{\text {eff }}$ between 5400 and $5600 \mathrm{~K}$ Be depletion is seen only among old field stars, while for temperatures below $5400 \mathrm{~K}$, Be depletion is seen also among the Hyades, but not among NGC 2516 members. This implies that the age at which Be depletion begins in cool stars increases with increasing $T_{\text {eff }}$ : namely, stars cooler than $\sim 5400 \mathrm{~K}$ start depleting Be between 150 and $600 \mathrm{Myr}$, depletion starts after 600 Myr for stars in the 5400-5600 interval, while stars

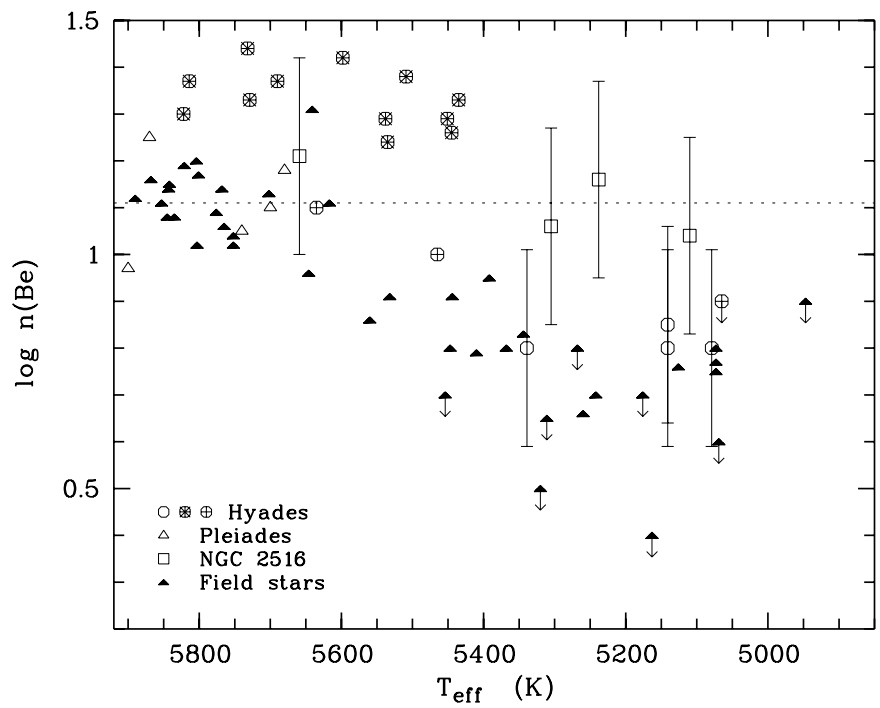

Fig. 7. $\log n(\mathrm{Be})$ as a function of $T_{\text {eff }}$ for NGC $2516(150 \mathrm{Myr}-$ open squares), the Hyades (600 Myr - present sample: open circles; sample of Boesgaard et al. 2004a: open circles with stars; sample of García López et al. 1995: open circles with plus symbols), Pleiades (125 Myr - open triangles; data of Boesgaard et al. 2003), and field stars (>1 Gyr - filled triangles; sample of Santos et al. 2004). The horizontal line denotes the initial Be abundance in our scale.

warmer than $5600 \mathrm{~K}$ (but cooler than $6150 \mathrm{~K}$ ), including the Sun, never deplete Be.

Li depletion in MS stars cooler than $5500-5400 \mathrm{~K}$ is normally explained with convection, since the convective envelope reaches deep enough to mix $\mathrm{Li}$-poor material into the surface. The question then arises whether Be depletion is also expected for these stars based on standard models. In order to address this question, we calculated evolutionary tracks for a $0.9 M_{\odot}$ star, corresponding to the mass of our coolest stars in the Hyades (see below), using the models by Sestito et al. (2006). These were computed employing an updated version of the evolutionary code FRANEC (e.g. Chieffi \& Straniero 1989) and adopting updated physical inputs (equation of state, opacity tables, nuclear cross sections, etc., see Sestito et al. 2006, for further details). As customary in standard models, the only mixing mechanism included is convection, whose extension was determined by adopting the classical Schwarzschild criterion and the mixing length formalism. Element diffusion has been included during the MS (Ciacio et al. 1997; with diffusion coefficients from Thoul et al. 1994). Three different models were computed; namely, we adopted two different solar mixtures: the solar composition by Asplund et al. (2004; hereafter As04) and that by Grevesse \& Noels (1993; hereafter GN93). The first track computed is the "standard" model by Sestito et al. (2006; model 1 in their Table 1): As04 mixture, $Z=0.013$ (corresponding to $[\mathrm{Fe} / \mathrm{H}]=0$ for this composition), He content $Y=0.27$, and mixing length parameter $\alpha=1.9$. The other two models have the same He abundance and $\alpha$ but the Fe content of the Hyades $([\mathrm{Fe} / \mathrm{H}]=+0.13)$ has been considered, in one case adopting the As04 mixture (and the resulting global metallicity is $Z=0.018$ ), while in the other using the GN93 composition $(Z=0.025)$. We mention that at the age of the Hyades, the [As04, $Z=0.013$ ] model has an effective temperature of $5400 \mathrm{~K}$, while the [As04, $Z=0.018]$ model has $T_{\text {eff }}=5200 \mathrm{~K}$ and the [GN93, $\left.Z=0.025\right]$ one has $T_{\text {eff }}=5130 \mathrm{~K}$, consistent with the temperatures of Hyades stars in our sample. 


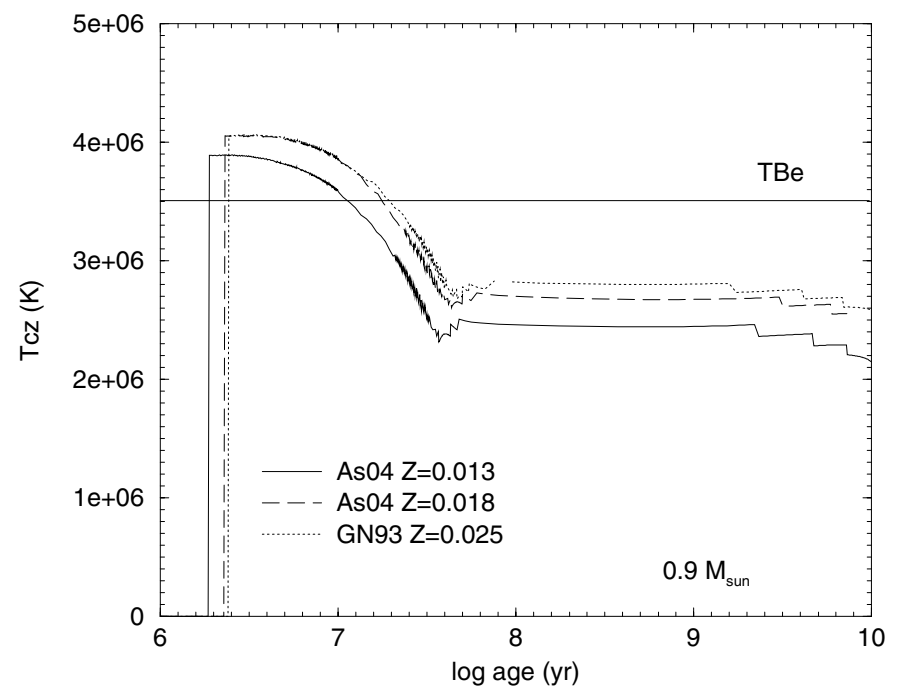

Fig. 8. Model predictions for the temperature at the base of the convective zone as a function of age. The solid horizontal line indicates the temperature necessary for Be to burn; the solid, dashed, and dotted curves show the predictions of the three different models described in the text.

In Fig. 8 we plot the temperature at the base of the convective envelope $\left(T_{\mathrm{CZ}}\right)$ predicted by our models as a function of time: the figure clearly shows that, for all the models, $T_{\mathrm{CZ}}$ is slightly higher than the Be ignition temperature only for ages younger than about $20 \mathrm{Myr}$, implying that Be depletion can in principle occur only during PMS, while no Be depletion is expected at older ages. This is shown in Fig. 9 where we plot the resulting evolution of $\mathrm{Be} / \mathrm{Be}_{0}$ as a function of age: consistently with the discussion above, none of the three models predict significant Be depletion. The model with solar metallicity predicts a slightly lower amount of Be depletion with respect to the other two, as expected (the higher is $Z$, the higher is the efficiency of convection). However, and most importantly, the difference between the solar metallicity track and the two models with the Fe content of the Hyades is almost negligible, and in all cases a very small amount of $\mathrm{Be}$ is depleted with respect to the initial value. In particular, we note that even PMS Be depletion is always very small (almost negligible), since $T_{\mathrm{CZ}}$ reaches values only slightly higher than the temperature of Be ignition and, furthermore, the exact value of ignition temperature depends on the physical assumptions in the code. We mention in passing that this result is in agreement with the early calculations of Bodenheimer (1966).

Our conclusion is that Be depletion observed in the cool Hyades with respect to NGC 2516 cannot be explained only by convective mixing and cannot be reproduced in the framework of standard models. An additional, or extra-mixing mechanism must be present, analogously to what invoked to explain Li depletion in more massive, warmer stars. Most obviously, the mechanism is not necessarily the same. Our Be determinations suggest that this physical process starts being efficient after an age of $\sim 150 \mathrm{Myr}$.

\subsection{Stars out of the main sequence}

\subsubsection{Subgiants}

M 67 subgiants S1034 and S1239 studied here were also included in the Li survey of Balachandran (1995), who derived for the two stars masses and ZAMS temperatures equal to $1.282 M_{\odot}$ and $6349 \mathrm{~K}$ (S1034) and $1.3 M_{\odot}$ and $6450 \mathrm{~K}$ (S1239); as

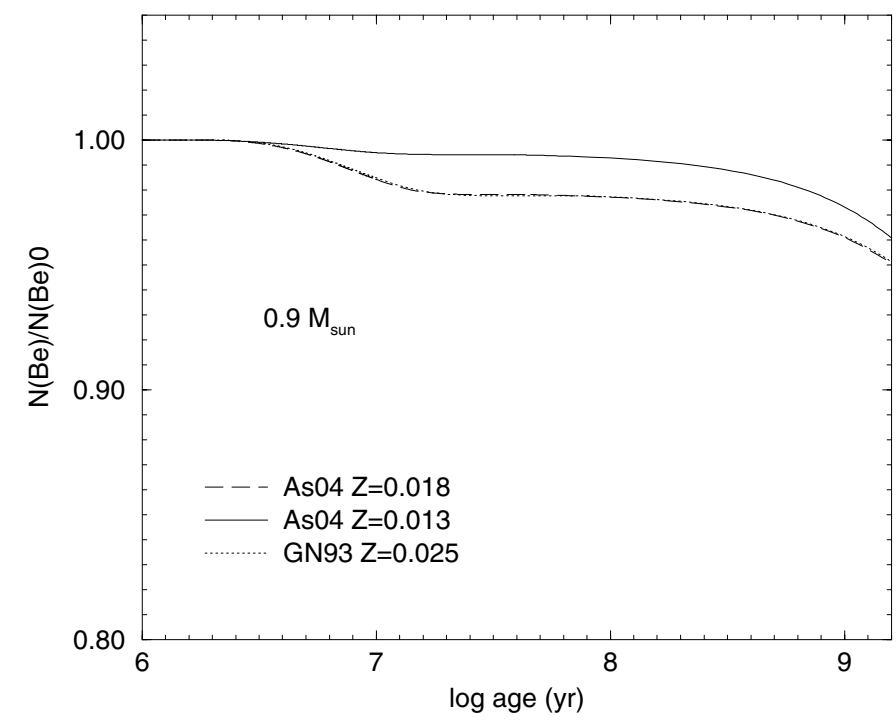

Fig. 9. Model predictions for the evolution of $N(\mathrm{Be}) / N(\mathrm{Be})_{0}$ as a function of age for the three different models.

argue by Balachandran, their low Li abundances strongly support the hypothesis that they have depleted Li during the MS phases and have evolved from the Li gap region. Our study provides the first measurements of Be in stars evolved from the gap, thus allowing us to carry out further comparison with model predictions. Both stars show similar factors of Be depletion (a factor of about 10 - see Table 2), suggesting the presence of a Be gap, similarly to what found for the Hyades MS stars by Boesgaard \& King (2002). As discussed at length in the series of papers by Boesgaard and collaborators and mentioned in Sect. 1 the tight correlation between $\mathrm{Be}$ and $\mathrm{Li}$ depletion for stars in the gap suggests that light element depletion is driven by rotational mixing. Our Be determinations for the two subgiants are consistent with this hypothesis.

More quantitatively, Sills \& Deliyannis (2000) presented evolutionary models of subgiants in M 67 including three mixing processes (diffusion, mass loss, rotational mixing) and following the evolution of both $\mathrm{Li}$ and $\mathrm{Be}$ abundances. They concluded that Li measurements are in good agreement with models including mixing driven by rotation; they stressed however that Be data for subgiants in M 67 would also be very helpful in order to constrain the various free parameters entering the models. Our measurements of Be for two subgiants in M 67 represent a first step towards that direction. In Fig. 10 we compare our results with the predictions of the model of Sills \& Deliyannis including rotationally induced mixing; the other models discussed in that paper are not shown, since they were already ruled out the basis of $\mathrm{Li}$ alone. Note that the $T_{\text {eff }}$ sequence in the figure represents an evolutionary sequence for a given mass $\left(1.26 M_{\odot}\right.$ - somewhat below the value given by Balachandran 1995, for subgiants in M 67) rather than a sequence of different masses. The figure evidences a very good agreement between the model and our measurement for S1239, the cooler, slightly more massive and more evolved star, whose Be content is the result of MS depletion plus post-MS dilution. The agreement is considerably worse for the less evolved S1034, whose Be content is the result of MS depletion only, and which appears more Be depleted than predicted (but also its $\mathrm{Li}$ abundance is below model predictions - see Fig. 4 in Sills \& Deliyannis). We suggest that the reason for this discrepancy could be an initial higher rotational velocity for this star than the one assumed by 


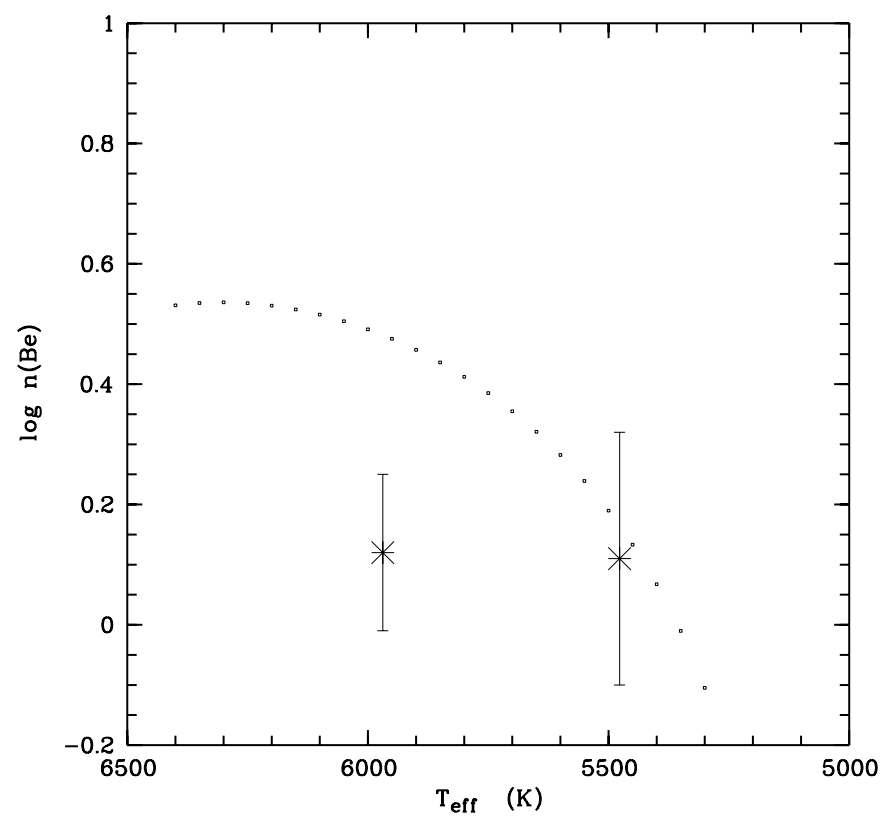

Fig. 10. Comparison of model predictions of $\log n(\mathrm{Be})$ for subgiant stars in M 67 with our measurements for S1034 and S1239. The figure is based on Fig. 6 in Sills \& Deliyannis (2000), but the initial Be abundance has been scaled to our value. With respect to that figure we show only the model including rotationally driven mixing.

Sills \& Deliyannis in their model $\left(v_{\text {in. }}=10 \mathrm{~km} \mathrm{~s}^{-1}\right)$, which would result in a larger amount of light element depletion on the MS.

\subsubsection{Blue stragglers}

Light elements also provide an useful tool to investigate the mechanism of formation of blue stragglers and, in particular, whether they are the product of collision processes or binary mergers. To our knowledge, no quantitative models exist to-date presenting predictions for $\mathrm{Li}$ and $\mathrm{Be}$ abundances for the different scenarii of blue straggler formation. However, Sills et al. (1997) qualitatively suggested that in the case a blue straggler is the result of a binary merger, it should not have observable $\mathrm{Li}$; on the other hand, collisions would not result in any $\mathrm{Li}$ (and a fortiori $\mathrm{Be}$ ) destruction.

The first observations of light elements in blue stragglers belonging to M 67 were performed by Pritchet \& Glaspey (1991), who measured Li in seven stars; they did not detect the Li line in any of them and concluded that some form of mixing, possibly due to binary coalescence, had affected the outer envelope of blue stragglers. We note however, that upper limits for most of their sample stars were indeed consistent with abundances of Li-poor MS cluster stars.

In a more recent study, Shetrone \& Sandquist (2000) carried out new Li measurements of blue stragglers in M 67, including both S997 and S2204, the two stars studied here. We refer the reader to Shetrone \& Sandquist (2000) for a detailed discussion of the properties of the two stars; we just recall that the high eccentricity binary nature of one of the two (S997) suggests that it is unlikely a binary merger, which is instead the most plausible scenario for the formation of S2204. Shetrone \& Sandquist (2000) did not detect the Li line in either star; although the nondetection of $\mathrm{Li}$ might favour the merger scenario, these authors point out that their upper limits eventually do not provide any clues on the formation mechanism. The upper limits are not very stringent and are consistent with the abundances of
MS unevolved cluster stars (i.e., the presence of Li cannot be ruled out); furthermore, even a low $\mathrm{Li}$ abundance could be explained by the fact that the two stars fall in the Li gap $T_{\text {eff }}$ interval and thus could have undergone significant depletion after the blue straggler is formed in the collision scenario.

Until the present study no Be determinations for blue stragglers in open clusters were available in the literature. We have now determined Be abundance for S997 and S2204, finding that they are both depleted, but affected by different amounts of Be depletion; namely, a factor of about 6 and 20 for S997 and S2204, respectively. At variance with $\mathrm{Li}$, the low Be abundances are not consistent with those of MS stars in M 67; as in the case of $\mathrm{Li}$, we cannot completely exclude that the two stars have depleted $\mathrm{Be}$ after formation. Nevertheless, we notice that Be abundance for star S2204 is much below the values measured for stars in the Be gap. Thus, whereas Be abundance in S997 would not be inconsistent with either scenario, we suggest that the factor of three higher depletion measured in S2204 might be an indication of a different formation of the two systems, with this star being the product of a binary merger.

\section{Conclusions}

We have carried out Be observations in three open clusters of different ages: NGC 2516, the Hyades, and M 67. The present study, together with available data from R02 for M 67 and from other previous Be surveys of open clusters, allows us to cover a grid of MS stars which span from $150 \mathrm{Myr}$ to $4.5 \mathrm{Gyr}$ in age and from the Li gap down to $5100 \mathrm{~K}$ in effective temperature.

We confirm that stars in different $T_{\text {eff }}$ interval are characterized by different amounts of Be depletion and Be vs. Li behaviors; namely, i. stars warmer than $6150 \mathrm{~K}$ are characterized by some Be depletion and show a Be vs. Li correlation; ii. Li and Be are instead not correlated for solar-type stars. Whereas Li depletion ranges between null (for the young star in IC 2391) and a factor larger than 100 (in the Sun and the Li poor stars in M 67), none of the stars show significant Be depletion; iii. the Hyades stars cooler than $5400 \mathrm{~K}$ are a factor of $\sim 1.8$ more Li depleted than stars of similar temperature in the younger NGC 2516; iv. subgiants and blue stragglers are severely depleted in Be.

The result for warm stars is consistent with the extensive work carried out by Boesgaard and collaborators and, in particular, with the observed Li-Be correlation for stars in the Li gap in the Hyades and other clusters of similar age. As mentioned in Sect. 1, the presence and slope of this correlation are in agreement with the predictions of models including slow mixing driven by rotation. Be depletion in the two subgiants in M 67 that are evolved from stars in the gap is also consistent with rotationally induced mixing and our measurements actually can provide additional constraints on theoretical models. On the contrary, the lack of a correlation between $\mathrm{Li}$ and $\mathrm{Be}$ for solar-type stars implies that the main process at work in these stars must be different. R02 indeed ruled out models including mixing induced by rotation and diffusion which predict a Be-Li correlation. They noticed that models including gravity waves would well fit the observed Be vs. Li distribution, but those models would not be able to explain the observed scatter in Li in M 67.

In a more realistic approach one might imagine that various processes (rotationally driven mixing, meridional circulation, diffusion, gravity waves, tachocline) are all present at the same time; different mechanisms might then either become dominant or counter-balance in different temperature regimes. In this context, very recently Charbonnel \& Talon (2005) presented a model including both mixing driven by rotation and gravity 
waves. They showed that this model is able to reproduce the solar $\mathrm{Li}$ abundance as well as the internal rotational profile of the Sun and that models with different initial rotational velocities would result in a range of $\mathrm{Li}$ abundances, consistent with the observed scatter in M 67. Since Charbonnel \& Talon (2005) did not provide any predictions for the evolution of Be abundances, we cannot make any quantitative comparison with the results of the present study. We also mention in passing that their model would not be able to reproduce the plateau in $\mathrm{Li}$ abundances that is observed for ages greater than 2 Gyr (Randich 2006, and references therein). More work is therefore needed on theoretical grounds; we stress however that considering the interplay between different processes might indeed represent the way towards the solution to the light element depletion problem.

As to cooler stars, whereas between $5600 \mathrm{~K}$ and $5400 \mathrm{~K}$ Be depletion is seen only in field stars older than $1 \mathrm{Gyr}$, when we reach stars as cool as $5400 \mathrm{~K}$, Be depletion starts at younger ages, between 150 and $600 \mathrm{Myr}$. We have shown that this cannot be explained by the effect of increasing depth of the convection zone (that instead explains Li depletion), and that an additional source of extra-mixing is needed also for cool stars. No models including extra-mixing processes are so far available for stars significantly cooler than the Sun; our results clearly point toward the necessity of developing such models.

Finally, our Be determination for the two M 67 blue stragglers does not allow us to draw any definitive conclusion on their formation scenario; nevertheless, the significant difference in their Be content suggests that they have formed by two different processes.

Acknowledgements. This research has made use of the SIMBAD data base, operated at CDS, Strasbourg, France. We thank the anonymous referee for the very useful comments and suggestions.

\section{References}

Anders, R., \& Grevesse, N. 1989, Geochim. Cosmochim. Acta, 53, 197 Asplund, M., Grevesse, N., Sauval, A. J., Allende Prieto, C., \& Kiselman, D. 2004, A\&A, 417, 751

Asplund, M., Grevesse, N., \& Sauval, A. J. 2005, in Cosmic Abundances as Records of Stellar Evolution and Nucleosynthesis, ed. T. G. Barnes III, \& F. N. Bash, ASP Conf. Ser., 336, 25

Balachandran, S. C. 1995, ApJ, 446, 203

Balachandran, S. C., \& Bell, R. A. 1998, Nature, 392, 791

Bodenheimer, P. 1966, ApJ, 144, 103

Boesgaard, A. M., \& Tripicco, M. J. 1986, ApJ, 302, L49

Boesgaard, A. M., \& King, J. R. 2002, ApJ, 565, 587

Boesgaard, A. M., Budge, K. G., \& Ramsay, M. E. 1988, ApJ, 327, 389

Boesgaard, A. M., Armengaud, E., \& King, J. R. 2003, ApJ, 582, 410

Boesgaard, A. M., Armengaud, E., \& King, J. R. 2004a, ApJ, 605, 864

Boesgaard, A. M., Armengaud, E., King., J. R., Deliyannis, C. P., \& Stephens, A. 2004 b, ApJ, 613, 1202
Brun, A. S., Turck-Chièze, S., \& Zahn, J.-P. 1999, ASP Conf. Ser., 173, 173 Chaboyer, B., Demarque, P., \& Pinsonneault, M. H. 1995, ApJ, 441, 865 Charbonnel, C., \& Talon, S. 1999, A\&A, 351, 635

Charbonnel, C., \& Primas, F. 2005, A\&A, 442, 961

Charbonnel, C., \& Talon, S. 2005, Science, 309, 2189

Chieffi, A., \& Straniero, O. 1989, ApJS, 71, 47

Chmielewski, Y., Müller, E. A., \& Brault, J. W. 1975, A\&A, 42, 37

Ciacio, F., Degl'Innocenti, S., \& Ricci, B. 1997, A\&AS, 123, 449

Dekker, H., D’ Odorico, S., Kaufer, A., Delabre, B., \& Kotzslowski, H. 2000, Proc. SPIE, 4008, 534

Deliyannis, C. P., \& Pinsonneault, M. 1997, ApJ, 488, 833

Deliyannis, C. P., Boesgaard, A. M., Stephens, A., et al. 1998, ApJ, 498, L147

García López, R. J., \& Spruit, H. C. 1991, ApJ, 377, 268

García López, R. J., Rebolo, R., \& Beckmann, J. E. 1988, PASP, 100, 1489

García López, R. J., Rebolo, R., \& Perez de Taoro, M. R. 1995, A\&A, 302, 184

Grevesse, N., \& Noels, A. 1993, in Origin and Evolution of the Elements, ed. N. Prantzos, E. Vangioni-Flam, \& M. Cassé (Cambridge: Cambrdige University Press), 15

Jeffries, R. D., James, D. J., \& Thurston, M. R. 1998, MNRAS, 300, 550

Jones, B. F., Fisher, D., \& Soderblom, D. R. 1999, AJ, 117, 330

Kurucz, R. L. 1993, CD-ROMs \#1, 13, 18

Michaud, G. 1986, ApJ, 302, 650

Montalbán, J., \& Schatzmann, E. 2000, A\&A, 354, 943

Nissen, P. E. 1981, A\&A, 97, 145

Pasquini, L., Randich, S., \& Pallavicini, R. 1997, A\&A, 325, 535

Piau, L., Randich, S., \& Palla, F. 2003, A\&A, 408, 1037

Pilachowski, C. A., Booth, J., \& Hobbs, L. M. 1987, PASP, 99, 1288

Pinsonneault, M. H., Deliyannis, C. P., \& Demarque, P. 1992, ApJS, 78, 179

Primas, F., Duncan, D. K., Pinsonneault, M. H., Deliyannis, C. P., \& Thorburn, J. A. 1997, ApJ, 480, 78

Pritchet, C. J., \& Glaspey, J. W. 1991, ApJ, 373, 105

Randich, S. 2006, in Chemical Abundances and Mixing in Stars in the Milky Way and its Satellites, ed. S. Randich, \& L. Pasquini (Springer-Verlag), 173

Randich, S., Pallavicini, R., Meola, G., Stauffer, J. R., \& Balachandran, S. 2001, A\&A, 372, 862

Randich, S., Primas, F., Pasquini, L., \& Pallavicini, R. 2002, A\&A, 387, 222 (R02)

Randich, S., Sestito, P., Primas, F., Pallavicini, R., \& Pasquini, L. 2006, A\&A, 450,557

Sanders, W. L. 1977, A\&AS, 27, 89

Santos, N. C., Israelian, G., Randich, S., García López, R. J., \& Rebolo, R. 2004, A\&A, 425, 1013

Sestito, P., degl' Innocenti, S., Prada Moroni, P., \& Randich, S. 2006, A\&A, 454, 311

Schatzman, E., \& Baglin, A. 1991, A\&A, 249, 125

Shetrone, M. D., \& Sandquist, E. L. 2000, AJ, 120, 1913

Sills, A., \& Deliyannis, C. P. 2000, ApJ, 544, 944

Sills, A., Lombardi, J. C. Jr., Bailyn, C. D., et al. 1997, ApJ, 487, 290

Soderblom, D. R., Stauffer, J. R., Hudon, J. D., \& Jones, B. F. 1993, ApJS, 85, 313

Spite, F., Spite, M., \& Peterson, R. C., Chaffee, F. H., Jr. 1987, A\&A, 171, L8

Steinhauer, A., \& Deliyannis, C. P. 2004, ApJ, 614, L65

Swenson, F. J., \& Faulkner, J. 1992, ApJ, 395, 654

Thorburn, J. A., Hobbs, L. M., Deliyannis, C. P., \& Pinsonneault, M. H. 1993, ApJ, 415, 150

Thoul, A. A., Bahcall, J. N., \& Loeb, A. 1994, ApJ, 421, 828

van Bueren, H. G. 1952, Bull. astron. Inst. Netherlands, 11, 385 